\title{
ON THE CONNECTIVITY OF THE BRANCH LOCUS OF THE SCHOTTKY SPACE
}

\author{
Rubén A. Hidalgo and Milagros Izquierdo \\ Universidad Técnica Federico Santa María, Departamento de Matemática \\ Casilla 110-V Valparaiso, Chile; ruben.hidalgo@usm.cl \\ Linköpings Universitet, Mathematiska Institutionen \\ 58183 Linköping, Sweden; milagros.izquierdo@liu.se
}

\begin{abstract}
Let $M$ be a handlebody of genus $g \geq 2$. The space $\mathcal{T}(M)$, that parametrizes marked Kleinian structures on $M$ up to isomorphisms, can be identified with the space $\mathcal{M} S_{g}$ of marked Schottky groups of rank $g$, so it carries a structure of complex manifold of finite dimension $3(g-1)$. The space $\mathcal{M}(M)$ parametrizing Kleinian structures on $M$ up to isomorphisms, can be identified with $\mathcal{S}_{g}$, the Schottky space of rank $g$, and it carries the structure of a complex orbifold. In these identifications, the projection map $\pi: \mathcal{T}(M) \rightarrow \mathcal{M}(M)$ corresponds to the map from $\mathcal{M} S_{g}$ onto $\mathcal{S}_{g}$ that forgets the marking. In this paper we observe that the singular locus $\mathcal{B}(M)$ of $\mathcal{M}(M)$, that is, the branch locus of $\pi$, has (i) exactly two connected components for $g=2$, (ii) at most two connected components for $g \geq 4$ even, and (iii) $\mathcal{M}(M)$ is connected for $g \geq 3$ odd.
\end{abstract}

\section{Introduction}

The conformal automorphisms of the Riemann sphere $\widehat{\mathbf{C}}$ are given by the Möbius transformations. By Poincare's extension theorem, each Möbius transformation extends to an orientation-preserving isometry of the hyperbolic 3-space $\mathbf{H}^{3}$ and, in fact, every orientation-preserving isometry of $\mathbf{H}^{3}$ is obtained in that way. We denote by $\mathbf{M} \cong \mathrm{PSL}_{2}(\mathbf{C})$ the group of Möbius transformations.

A Kleinian group is a discrete subgroup $\Gamma$ of $\mathbf{M}$ and its region of discontinuity is the (open) set $\Omega$ consisting of the points on $\widehat{\mathbf{C}}$ on which $\Gamma$ acts discontinuously (which might be empty). The quotient space $M_{\Gamma}=\left(\mathbf{H}^{3} \cup \Omega\right) / \Gamma$ (respectively, $S_{\Gamma}=\Omega / \Gamma$ ) is called the Kleinian orbifold (respectively, the Riemann orbifold) uniformized by $\Gamma$; $S_{\Gamma}$ is also called the conformal boundary of $M_{\Gamma}$. If $\Gamma$ is a torsion free Kleinian group, then $M_{\Gamma}$ is a manifold with conformal boundary the Riemann surface $\Omega / \Gamma$, interior the hyperbolic manifold $M_{\Gamma}^{0}=\mathbf{H}^{3} / \Gamma$, and $\pi_{1}(M)$ is isomorphic to $\Gamma$. A Kleinian structure on an orientable 3-manifold $M$ (possible with non-empty boundary) is given by a Kleinian group $\Gamma$ so that $M_{\Gamma}$ is (orientable-preserving) homeomorphic to $M$.

We assume from now on that $M$ is an oriented handlebody of genus $g$.

A Kleinian structure on the handlebody $M$ is provided by a Schottky group $\Gamma$ of rank $g$. In this case the conformal boundary $S_{\Gamma}$ is homeomorphic to the topological boundary $S$ of $M$ (that is, $\Gamma$ provides a Riemann surface structure to $S$ ).

doi:10.5186/aasfm.2014.3942

2010 Mathematics Subject Classification: Primary 30F10, 30F40.

Key words: Moduli space, branch locus, Schottky space, Schottky group, handlebody, Riemann surface, Kleinian group, Fuchsian group.

Partially supported by project Fondecyt 1110001 and UTFSM 12.13.01. 
A one-to-one homomorphism $\phi: \pi_{1}(M) \rightarrow \mathbf{M}$ will be called admissible for $M$ if $\phi\left(\pi_{1}\right)$ is a Schottky group providing a Kleinian structure on $M$.

Two admissible homomorphisms for $M$, say $\phi_{1}$ and $\phi_{2}$, will be called equivalent if there is some Möbius transformation $A \in \mathbf{M}$ so that $\phi_{2}(\alpha)=A \circ \phi_{1}(\alpha) \circ A^{-1}$, for every $\alpha \in \pi_{1}(M)$.

The set $\mathcal{T}(M)$ of equivalence classes of admissible homomorphisms for the handlebody $M$ is called the Teichmüller space of $M$. Classical quasiconformal deformation theory [26] asserts that $\mathcal{T}(M)$ carries the structure of a complex manifold of finite dimension. More precisely, if $\phi$ is a fixed admissible homomorphism for $M$, then $\mathcal{T}(M)$ can be identified with the quasiconformal deformation space of the Schottky group $\phi\left(\pi_{1}(M)\right)$.

Two Kleinian structures on the handlebody $M$, provided by Schottky groups $\Gamma_{1}$ and $\Gamma_{2}$, are called conformally equivalent if there is an orientation-preserving diffeomorphism $f: M_{\Gamma_{1}} \rightarrow M_{\Gamma_{2}}$ whose restriction $f: M_{\Gamma_{1}}^{0} \rightarrow M_{\Gamma_{2}}^{0}$ is an isometry (equivalently, the restriction $f: S_{\Gamma_{1}} \rightarrow S_{\Gamma_{2}}$ is a conformal homeomorphism). The moduli space of $M$, denoted by $\mathcal{M}(M)$, is the set of classes of conformally equivalent Kleinian structures on $M$.

If $\psi, \phi: \pi_{1}(M) \rightarrow \mathbf{M}$ are admissible homomorphisms for the handlebody $M$ defining the same class in $\mathcal{T}(M)$, then $\phi\left(\pi_{1}(M)\right)$ and $\psi\left(\pi_{1}(M)\right)$ define the same class in $\mathcal{M}(M)$. This fact asserts the existence of a natural (surjective) map $\pi: \mathcal{T}(M) \rightarrow$ $\mathcal{M}(M)$.

Let $\operatorname{Diff}^{+}(M)$ be the group of orientation-preserving self-diffeomorphisms of $M$ and let $\operatorname{Diff}_{0}(M)$ be its normal subgroup consisting of those diffeomorphisms isotopic to the identity. Then the modular group $\operatorname{Mod}(M)=\operatorname{Diff}^{+}(M) / \operatorname{Diff}_{0}(M)$ acts naturally on $\mathcal{T}(M)$ as a discrete group of holomorphic automorphisms [26]. It follows from the definition that the quotient space $\mathcal{T}(M) / \operatorname{Mod}(M)$ is a model for $\mathcal{M}(M)$ and that it is a finite dimensional orbifold. The projection $\pi: \mathcal{T}(M) \rightarrow \mathcal{M}(M)$ is then a regular branched covering with branch locus $\mathcal{B}(M)$. A goal is to study the topology of $\mathcal{B}(M)$, in particular, to study the connectedness of such a branch locus. Our main result is the following.

Theorem 1. If $M$ is a handlebody of genus $g \geq 2$, then (i) $\mathcal{B}(M)$ has at most two connected components for $g \geq 4$ even, (ii) $\mathcal{B}(M)$ has exactly two connected components if $g=2$ and (iii) if $g \geq 3$ is odd, then $\mathcal{B}(M)$ is connected.

We will see that $\mathcal{B}(M)$ consists of the union of two (not necessarily disjoint) connected sets, say $A$ and $B$. The points in $A$ corresponds to those Kleinian structures on $M$ admitting an automorphism of order two where the connected components of its fixed point set are an even number of simple loops and some simple arcs. The points in $B$ corresponds to those Kleinian structures on $M$ admitting an automorphism of order two where the connected components of its fixed point set are an odd number of simple loops and some simple arcs. In the case $g=2$ we will see that these two sets are disjoint and, for $g \geq 3$ odd, that they intersect. In the case $g \geq 4$ even we do not know if these two sets are disjoint or not, but we expect them to be disjoint. In order to see if these two sets $A$ and $B$ are disjoint, we need to prove that there is not a Kleinian structure on $M$ admitting two automorphisms of order two, say $\phi_{1}$ and $\phi_{2}$, so that the number of loops of fixed points of them are of different parity. Let us observe that, in case the above is possible, the group $G=\left\langle\phi_{1}, \phi_{2}\right\rangle$ is isomorphic to a dihedral group or order $2 N$. Clearly $N$ has to be even (otherwise 
the two involutions are conjugated in $G$, their fixed point sets have the same number of connected components which are loops, a contradiction). If $N \equiv 2(\bmod 4)$, it is possible to find in $G$ another involution, say $\phi_{3}$, which is conjugate to $\phi_{2}$ and so that $\left\langle\phi_{1}, \phi_{3}\right\rangle$ is isomorphic to the abelian group $\mathbf{Z}_{2}^{2}$.

\section{An interpretation in terms of Schottky groups}

In this section we describe with some detail the identifications above, in terms of Schottky groups. In particular, we reinterpret Theorem 1 in these terms (our proof will be done in these terms).

2.1. Kleinian groups. A Kleinian group is a discrete subgroup of the group of Möbius transformations $\mathbf{M} \cong \mathrm{PSL}_{2}(\mathbf{C})$; the group of conformal automorphisms of the Riemann sphere $\widehat{\mathbf{C}}$. Each Möbius transformation extends naturally to an isometry of the hyperbolic space $\mathbf{H}^{3}$ as an orientation-preserving isometry.

If $K$ is a Kleinian group and $p \in \widehat{\mathbf{C}}$, then we say that $K$ acts discontinuously on $p$ if its $K$-stabilizer $K_{p}=\{A \in K: A(p)=p\}$ is finite and there is an open set $U \subset \widehat{\mathbf{C}}$ such that $p \in U$ and $A(U) \cap U=\emptyset$ for $A \in K-K_{p}$. The region of discontinuity of $K$ is the set $\Omega$ consisting of all the points over which $K$ acts discontinuosly. The book [23] is a good reference on the theory of Klenian groups.

2.2. Schottky groups. A Schottky group of rank 0 is just the trivial group. A Schottky group of rank $g \geq 1$ is a Kleinian group $\Gamma$ which is isomorphic to a free group of rank $g$, all of its non-trivial elements are loxodromic and it has non-empty region of discontinuity.

A Schottky group of rank $g \geq 1$ can be constructed as follows. Let $C_{k}, C_{k}^{\prime}$, $k=1, \ldots, g$, be $2 g$ Jordan curves on the Riemann sphere $\widehat{\mathbf{C}}$ such that they are mutually disjoint and bound a $2 g$-connected domain, say $\mathcal{D}$. Suppose that for each $k$ there exists a fractional linear transformation $A_{k} \in \operatorname{PSL}(2, \mathbf{C})$ so that (i) $A_{k}\left(C_{k}\right)=C_{k}^{\prime}$ and (ii) $A_{k}(\mathcal{D}) \cap \mathcal{D}=\emptyset$. The group $\Gamma$, generated by all these transformations is a Schottky group of rank $g$. This is mainly a consequence of Klein-Maskit's combination theorems [22, 24].

That all Schottky groups are obtained from the above construction is mainly due to Chuckrow [9].

If $\Omega$ is the region of discontinuity of a Schottky group $\Gamma$, say of rank $g$, then it is known that $\Omega$ is connected, that $\Omega / \Gamma$ is a closed Riemann surface of genus $g$ and that $\left(\mathbf{H}^{3} \cup \Omega\right) / \Gamma$ is a handlebody of genus $g$.

2.3. Schottky space. Two Schottky groups of the same rank $g$, say $\Gamma_{1}$ and $\Gamma_{2}$, are conjugate if there is a Möbius transformation $A$ so that $\Gamma_{2}=A \Gamma_{1} A^{-1}$. If $\Gamma$ is a Schottky group, then we denote by $[\Gamma]$ its conjugacy class. The space that parametrizes conjugacy classes of Schottky groups of rank $g$ is called the Schottky space of rank $g$, which we denote as $\mathcal{S}_{g}$.

2.4. Marked Schottky space. A marked Schottky group of rank $g$ is a tuple $\left(\Gamma, C_{1}, \ldots, C_{g}\right)$, where $\Gamma$ is a Schottky group of rank $g$ and $C_{1}, \ldots, C_{g}$ is a set of generators of $\Gamma$.

Two marked Schottky groups of rank $g$, say $\left(\Gamma, C_{1}, \ldots, C_{g}\right)$ and $\left(\widehat{\Gamma}, \widehat{C}_{1}, \ldots, \widehat{C}_{q}\right)$, are said to be equivalent if there is a Möbius transformation $A$ so that $A C_{j} A^{-1}=$ $\widehat{C}_{j}$, for every $j=1, \ldots, g$. We denote by $\left[\left(\Gamma, C_{1}, \ldots, C_{g}\right)\right]$ the equivalence class of 
$\left(\Gamma, C_{1}, \ldots, C_{g}\right)$. The space that parametrizes equivalence classes of marked Schottky groups of rank $g$ is called the marked Schottky space of rank $g$, which we denote as $\mathcal{M} S_{g}$.

We have that $\mathcal{M} S_{0}=\mathcal{S}_{0}$ consists of one point and that $\mathcal{M} S_{1}=\mathcal{S}_{1}$ can be identified with the punctured unit disc. If $g \geq 2$, then $\mathcal{M} S_{g}$ can be identified with the quasiconformal deformation space of a fixed Schottky group of rank $g$; so it is a complex manifold of dimension $3(g-1)[6,7,26]$ (see Section 3).

2.5. Identification of $\mathcal{T}(\boldsymbol{M})$ with $\mathcal{M} \boldsymbol{S}_{\boldsymbol{g}}$. We fix a set of generators $a_{1}, \ldots, a_{g}$ for the free group $\pi_{1}(M)$ of rank $g$.

To an admissible homomorphism $\phi: \pi_{1}(M) \rightarrow \mathbf{M}$ we may associated the marked Schottky group $\left(\Gamma, C_{1}, \ldots, C_{g}\right)$, where $C_{j}=\phi\left(a_{j}\right)$ and $\Gamma=\left\langle C_{1}, \ldots, C_{g}\right\rangle$; we also say that $\left(\Gamma, C_{1}, \ldots, C_{g}\right)$ is a marked Schottky structure on $M$.

Let $\phi$ and $\psi$ two admissible homomorphisms for $M$. Let us consider the corresponding marked Schottky structures, say $\left(\phi\left(\pi_{1}(M)\right)=\Gamma, \phi\left(a_{1}\right)=C_{1}, \ldots, \phi\left(a_{g}\right)=\right.$ $\left.C_{g}\right)$ and $\left(\psi\left(\pi_{1}(M)\right)=\widehat{\Gamma}, \psi\left(a_{1}\right)=\widehat{C}_{1}, \ldots, \psi\left(a_{g}\right)=\widehat{C}_{g}\right)$. By the definition, these two admissible homomorphisms are equivalent if and only if the corresponding marked Schottky groups are equivalent.

The above permits to observe that there is a natural bijection between $\mathcal{M} S_{g}$ and $\mathcal{T}(M)$, in particular, to provide a complex manifold structure on $\mathcal{T}(M)$.

2.6. Identification of $\mathcal{M}(\boldsymbol{M})$ with $\mathcal{S}_{\boldsymbol{g}}$. Now, if $\Gamma_{1}$ and $\Gamma_{2}$ are two Schottky groups providing equivalent Schottky structures on the handlebody $M$, then there is, by the definition, an orientation-preserving diffeomorphism $f: M_{\Gamma_{1}} \rightarrow M_{\Gamma_{2}}$, By lifting its restriction $f: M_{\Gamma_{1}}^{0} \rightarrow M_{\Gamma_{2}}^{0}$ to the universal cover, we obtain an orientationpreserving isometry $A$ (a Móbius transformation) of the hyperbolic space $\mathbf{H}^{3}$. As $A$ necessarily conjugates $\Gamma_{1}$ into $\Gamma_{2}$, we may see that $\left[\Gamma_{1}\right]=\left[\Gamma_{2}\right]$. The converse is clear. In this way we see that there is a natural bijection between $\mathcal{S}_{g}$ and $\mathcal{M}(M)$.

If $g \geq 2$, then $\mathcal{M} S_{g}$ can be identified with the quasiconformal deformation space of a fixed Schottky group of rank $g$; so it is a complex orbifold of dimension $3(g-1)$ $[6,7,26]$.

2.7. A description of $\pi: \mathcal{T}(M) \rightarrow \mathcal{M}(M)$. With the above identifications, the projection $\pi: \mathcal{T}(M) \rightarrow \mathcal{M}(M)$ corresponds to the map

$$
\pi: \mathcal{M} S_{g} \rightarrow \mathcal{S}_{g}, \quad\left[\left(\Gamma, C_{1}, \ldots, C_{g}\right)\right] \mapsto[\Gamma] .
$$

2.8. The modular group. The group of holomorphic automorphisms of $\mathcal{M} S_{g}$ is known to be isomorphic to the outer automorphism group $\operatorname{Out}\left(F_{g}\right)=$ $\operatorname{Aut}\left(F_{g}\right) / \operatorname{Inn}\left(F_{g}\right)$, where $F_{g}$ is the free group of rank $g$, and the above projection $\pi: \mathcal{M} S_{g} \rightarrow \mathcal{S}_{g}$ is a regular (branched) cover whose deck group is $\operatorname{Out}\left(F_{g}\right)$ [11]. In this way, we may identify the modular $\operatorname{group} \operatorname{Mod}(M)$ with the $\operatorname{group} \operatorname{Out}\left(F_{g}\right)$ of outer automorphisms of the free group of rank $g$. As the space $\mathcal{M}(M)$ is naturally identified (as seen above) to $\mathcal{S}_{g}$, and $\mathcal{S}_{g}=\mathcal{M} S_{g} / \operatorname{Out}\left(F_{g}\right)$, we see that $\mathcal{M}(M)$ carries a natural structure of a complex orbifold or analytic space.

2.9. An interpretation of Theorem 1 in terms of Schottky groups. Let $\mathcal{B}_{g}$ denote the locus of branch points of the quotient map $\pi: \mathcal{M} S_{g} \rightarrow \mathcal{S}_{g}$. Then, with all the above identifications, we have that $\mathcal{B}(M)$ is identified with $\mathcal{B}_{g}$.

We have that $\mathcal{M} S_{0}=\mathcal{S}_{0}$ consists of one point and that $\mathcal{M} S_{1}=\mathcal{S}_{1}$ can be identified with the punctured unit disc; in particular, $\mathcal{B}_{g}=\emptyset$ for $g \leq 1$. 
Theorem 1 can be now written as follows:

Theorem 2. The locus $\mathcal{B}_{2}$ has exactly two connected components. If $g \geq 4$ is even, then $\mathcal{B}_{g}$ has at most two connected components. If $g \geq 3$ is odd, then $\mathcal{B}_{g}$ is connected.

Remark 3. Let $M=M_{\Gamma}$ be a handlebody with a Schottky structure produced by a Schottky group $\Gamma$ of rank $g \geq 2$. The conformal boundary $S_{\Gamma}=\Omega / \Gamma$ of $M_{\Gamma}$ is a closed Riemann surface of genus $g$. There is a natural surjective holomorphic map $\tau: \mathcal{S}_{g} \rightarrow \mathcal{M}_{g}$, where $\mathcal{M}_{g}$ is the moduli space of closed Riemann surfaces of genus $g$. This map is a non-Galois branched covering through which the Galois branched covering $\widehat{\pi}: \mathcal{T}_{g} \rightarrow \mathcal{M}_{g}$ factors, where $\mathcal{T}_{g}$ is the Teichmüller space of closed Riemann surfaces of genus $g$. The connectivity of the branch locus of $\widehat{\pi}$ in $\mathcal{M}_{g}$ has been studied in $[4,3,2,5]$. In general the branch locus in $\mathcal{M}_{g}$ is disconnected and there are infinite families of genera where the number of connected components is an increasing function of the genus. This is clearly contrary to the result stated in Theorems 1 and 2. One of the main reasons for this to happen is that there are examples of closed Riemann surfaces $S$ admitting a conformal automorphism $f: S \rightarrow S$ which cannot be continuously extended as a self-homeomorphism of any handlebody $M$, with a Schottky structure, whose conformal boundary is $S$ [14]. For instance conformal automorphisms with $S /\langle f\rangle$ of genus zero and exactly three cone points.

\section{Quasiconformal deformation spaces of Kleinian groups}

In this section we review some definitions and classical results concerning quasiconformal deformation spaces of Kleinian groups. As we will be working with Kleinian groups containing a Schottky group as a finite index subgroup, these groups will be finitely generated with a connected region of discontinuity. For this reason, we will only recall the quasiconformal deformation theory for these types of Kleinian groups.

In particular, the marked Schottky space $\mathcal{S}_{g}$ will be seen as the quasiconformal deformation space of a Schottky group of rank $g$.

3.1. Quasiconformal homeomorphisms. Let $\Omega_{1}, \Omega_{2} \subset \widehat{\mathbf{C}}$ be non-empty domains. An orientation-preserving homeomorphism $W: \Omega_{1} \rightarrow \Omega_{2}$ is called a quasiconformal homeomorphism if it satisfies the following two conditions:

(i) $W$ has distributional partial derivatives with respect to $z$ and $\bar{z}$ which can be represented by locally integrable functions $W_{z}$ and $W_{\bar{z}}$, respectively, on $\Omega_{1}$;

(ii) there is a measurable function $\mu: \Omega_{1} \rightarrow \mathbf{C}$ (called a complex dilation of $W$ ) such that $\|\mu\|_{\infty}<1$, where \|\|$_{\infty}$ denotes the essential supreme norm, (that is, $\mu \in L_{1}^{\infty}\left(\Omega_{1}\right)$ ), and $W$ satisfies the Beltrami equation

$$
W_{\bar{z}}(z)=\mu(z) W_{z}(z) \quad \text { a.e. } z \in \Omega_{1} \text {. }
$$

The existence and uniqueness of quasiconformal homeomorphisms is due to Morrey [25].

Theorem 4. [25] If $\mu: \mathbf{C} \rightarrow \mathbf{C}$ is a measurable function with $\|\mu\|_{\infty}<1$, then there is a unique quasiconformal homeomorphism $W_{\mu}: \widehat{\mathbf{C}} \rightarrow \widehat{\mathbf{C}}$, with complex dilation $\mu$, satisfying

$$
W_{\mu}(\infty)=\infty, W_{\mu}(0)=0, W_{\mu}(1)=1
$$


The solution $W_{\mu}$ in the above theorem is called a normalized quasiconformal homeomorphism for $\mu$. Ahlfors-Bers [1] proved that the normalized quasiconformal homeomorphism varies continuously with $\mu$.

Theorem 5. (Measurable Riemann mapping's theorem [1]) With the notations of the previous theorem. If $\mu$ varies continuously (in the Banach space $L^{\infty}(\mathbf{C})$ ), then $W_{\mu}$ also varies locally uniformly continuously in the space of continuous maps on $\mathbf{C}$.

3.2. Quasiconformal deformation spaces. Let $K$ be a finitely generated Kleinian group with connected region of discontinuity $\Omega$. Associated to $K$ is the Banach space $L^{\infty}(K)$ (with the essential supreme norm \|\|$_{\infty}$ ) whose elements are those measurable functions $\mu: \widehat{\mathbf{C}} \rightarrow \mathbf{C}$ so that

$$
\begin{cases}\mu(z)=0, & \forall z \in \widehat{\mathbf{C}}-\Omega, \\ \mu(k(z)) \overline{k_{z}(z)}=k_{z}(z) \mu(z), & \text { if } z \in \Omega \text { and } k \in K .\end{cases}
$$

Let $L_{1}^{\infty}(K)$ be the open unit ball in $L^{\infty}(K)$; its elements are called the Beltrami coefficients of $K$.

By Theorem 4, for each Beltrami coefficient $\mu \in L_{1}^{\infty}(K)$ there is a unique quasiconformal homeomorphism $W_{\mu}: \widehat{\mathbf{C}} \rightarrow \widehat{\mathbf{C}}$, with complex dilation $\mu$, that fixes 0,1 and $\infty$. Now, for each $k \in K$, the element $k_{\mu}=W_{\mu} \circ k \circ W_{\mu}^{-1}$ is again a Möbius transformation. If we set $K_{\mu}=W_{\mu} K W_{\mu}^{-1}$, then the above provides an isomorphism of Kleinian groups $\chi_{\mu}: K \rightarrow K_{\mu}: k \mapsto k_{\mu}$ (the image $W_{\mu}(\Omega)$ is the region of discontinuity of $K_{\mu}$ ).

We say that $\mu_{1}, \mu_{2} \in L_{1}^{\infty}(K)$ are quasiconformal equivalent, denoted this by $\mu_{1} \sim \mu_{2}$, if $\chi_{\mu_{1}}=\chi_{\mu_{2}}$. If the group $K$ is non-elementary, then this is equivalent to say that $W_{\mu_{1}}$ and $W_{\mu_{2}}$ coincide at the limit set of $K$.

The quasiconformal deformation space of $K$ is the quotient space $\mathcal{Q}(K)=L_{1}^{\infty}(K)$ $/ \sim$. As a consequence of the measurable Riemann mapping's theorem, the space $\mathcal{Q}(K)$ is connected.

As the Kleinian group $K$ has been assumed to be finitely generated, it is well known that $\mathcal{Q}(K)$ is a complex manifold of finite dimension [21].

Remark 6. The above definitions can be done for finitely generated Kleinian groups $K$ with disconnected region of discontinuity with the property that $\Omega$ has a connected component $\Delta$ which is invariant under $K$. In such a case, one obtains the so called quasiconformal deformation space $\mathcal{Q}(K, \Delta)$. In that case, when $\Delta$ is simply connected the space $\mathcal{Q}(K, \Delta)$ is a model of the Teichmüller space of the orbifold $\Delta / K$ $[6,26]$.

3.3. Schottky space. If $\Gamma$ is a Schottky group of rank $g$, then by [7] its quasiconformal deformation space $\mathcal{Q}(\Gamma)$ turns out to be a connected complex manifold of dimension

$$
\operatorname{dim}_{\mathbf{C}} \mathcal{Q}(\Gamma)= \begin{cases}3 g-3, & g \geq 2 \\ 1, & g=1 \\ 0, & g=0\end{cases}
$$

As any two Schottky groups of the same rank $g$ are quasiconformally equivalent, their respective quasiconformal deformation spaces are complex analytically equivalent. It can be seen that if $\Gamma$ is a Schottky group of rank $g$, then $\mathcal{Q}(\Gamma)$ is isomorphic to $\mathcal{M} S_{g}$; that is $\mathcal{Q}(\Gamma)$ is a model of the marked Schottky space $\mathcal{M} S_{g}$. 
To obtain a model of $\mathcal{S}_{g}$, one has to consider the following equivalence relation on $\mathcal{Q}(\Gamma)$ : two deformations $\omega_{1}$ and $\omega_{2}$ are equivalent if there is a Möbius transformations $A$ so that $\omega_{1} \Gamma \omega_{1}^{-1}=A \omega_{2} \Gamma \omega_{2}^{-1} A^{-1}$. Then, the set of equivalence classes is a model for $\mathcal{S}_{g}$.

3.4. An embedding property. Let $\Gamma$ be a Schottky group of rank $g \geq 2$.

Let us first assume that there is a Kleinian group $K$ containing $\Gamma$ as a finite index normal subgroup (in particular, $K$ is finitely generated). As each Beltrami coefficient for $K$ is also a Beltrami differential for $\Gamma$ and both $K$ and $\Gamma$ have the same limit set, there is a natural holomorphic embedding

$$
\iota: \mathcal{Q}(K) \rightarrow \mathcal{Q}(\Gamma)
$$

so that $[0] \in \iota(\mathcal{Q}(K)) \subset \mathcal{Q}(\Gamma)$.

Now, let us assume that $\Gamma$ is not contained as a finite index normal subgroup of another Kleinian group. If there is some $[\mu] \in \mathcal{Q}(\Gamma)$ so that the Schottky group $\Gamma_{u}$ is contained in some Kleinian group $K$ as a finite index normal subgroup, then the above provides a holomorphic embedding

$$
j: \mathcal{Q}(K) \rightarrow \mathcal{Q}(\Gamma), \quad j([0])=[\mu] .
$$

\section{Cyclic-Schottky groups}

Our constructions of connected subsets of the branch locus $\mathcal{B}_{g}$ will be done in terms of certain Kleinian groups containing Schottky groups of rank $g$ as normal subgroups of finite index. It is known $[12,27]$ that, for $g \geq 2$, such an index is bounded above by $12(g-1)$.

4.1. Maximal Schottky extension groups. Let $K$ be a Kleinian group containing a Schottky group of rank $g \geq 2$ as a normal subgroup of maximal index $12(g-1)$. In this situation we say that $K$ is a maximal Schottky extension group. Maximal Schottky extension groups play the same role for handlebodies as the Fuchsian triangular group $(0 ; 2,3,7)$ plays for closed Riemann surfaces. There are exactly four types of isomorphic classes of maximal Schottky extension groups $K$ :

$$
D_{2} * \mathbf{z}_{2} D_{3}, D_{3} * \mathbf{z}_{3} \mathcal{A}_{4}, D_{4} * \mathbf{z}_{4} \mathfrak{S}_{4}, D_{5} * \mathbf{z}_{5} \mathcal{A}_{5},
$$

where $D_{r}$ is the dihedral group of order $2 r, \mathcal{A}_{r}$ is the alternating group in $r$ letters and $\mathfrak{S}_{4}$ is the symmetric group in 4 letters [27]. A description of these maximal Schottky extension groups, in terms of the Klein-Maskit combination theorems [22, 24] may be found in $[15,16]$.

Let $K$ be a maximal Schottky extension group. By definition, $K$ contains some Schottky group $\Gamma$ of rank $g \geq 2$ as a normal subgroup of index $12(g-1)$ (and any Schottky group of rank $g$ which is a normal subgroup of finite index of $K$ has index at most $12(g-1))$. We do not know if, for fixed $g$, such Schottky group is unique. Now, as seen above, there is an embedding $\iota: \mathcal{Q}(K) \rightarrow \mathcal{Q}(\Gamma)$ and $\pi(\iota(\mathcal{Q}(K)))$ is a 1-dimensional locus inside $\mathcal{B}_{g}$.

The 1-dimensional loci obtained as above inside $\mathcal{B}_{g}$ are necessarily disjoint for different algebraic types of $K$, but it may be that there is a connected component of $\mathcal{B}_{g}$ containing two of them.

In [16] we have constructed, for $g \in\{2,3,5,11\}$, explicit embeddings of quasiconformal deformations of these maximal Schottky extension groups into the marked Schottky space. The constructions are given by explicit surjective homomorphisms 
$\theta: K \rightarrow G$, where $G$ is either isomorphic to one of $D_{3}, \mathcal{A}_{4}, \mathcal{A}_{5}, \mathfrak{S}_{4}$, and $K \cong D_{2} * \mathbf{z}_{2} D_{3}$. The kernel $\Gamma$ of $\theta$ is a Schottky group as desired.

If we set $\Gamma^{2}=\left\langle\gamma^{2}: \gamma \in \Gamma\right\rangle$, then $\Gamma^{2}$ is a normal subgroup of $\Gamma$ of index $2^{g}$ in $\Gamma$. Thus $\Gamma^{2}$ is a Schottky group of rank $2^{g}(g-1)+1$, where $g$ is the rank of $\Gamma$, which is a normal subgroup of $K$. So, we obtain infinitely many genera with examples of such embeddings.

4.2. Cyclic extension of Schottky groups. A Kleinian group $K$ containing a Schottky group $\Gamma$ of rank $g$ as a finite index normal subgroup so that $K / \Gamma$ is a cyclic group is called a cyclic extension Schottky group. A geometrical picture of these Kleinian groups is provided in [13].

Below, we proceed to recall such a picture for the case that $K / \Gamma$ is a cyclic group of prime order $p$. In this case, the results in [13] state that $K$ is a free product, in the sense of the Klein-Maskit combination theorems (see Figure 1), of $t$ cyclic groups generated by loxodromic transformations, $r$ cyclic groups generated by elliptic transformations of order $p$ and $s$ Abelian groups, each one generated by a loxodromic transformation and an elliptic transformation of order $p$ both of them commuting, so that $g=1+p(t+r+s-1)-r$. In particular

$$
K \cong \mathbf{Z} * \cdots \cdot{ }^{t} * \mathbf{Z} * \mathbf{Z}_{p} * \cdots * \mathbf{Z}_{p} *\left(\mathbf{Z} \times \mathbf{Z}_{p}\right) * \cdots *\left(\mathbf{Z} \times \mathbf{Z}_{p}\right) .
$$

We say that $K$ is a cyclic-Schottky group of type $(g, p ; t, r, s)$. The above geometric structure permits us to see that (i) the fixed point set of $\phi$ in $M=\left(\mathbf{H}^{3} \cup \Omega\right) / \Gamma$ consists of $r$ simple arcs (with end points in the conformal boundary) and $s$ simple loops (all of them pairwise disjoint) and that (ii) the quotient orbifold $\mathcal{O}=M /\langle\phi\rangle$ is a closed 3-manifold homeomorphic to a handlebody of genus $t+s$. In this case, the branch locus of $\mathcal{O}$ is a collection of $r$ pairwise disjoint simple arcs and a collection of $s$ pairwise disjoint simple loops (also disjoint from the previous arcs). If $S=\Omega / \Gamma$, then $S$ is a closed Riemann surface of genus $g$ admitting a conformal automorphism $\phi$ of order $p$ with $S /\langle\phi\rangle$ of signature $(\gamma ; p, .2 r ., p)$.

The above description permits also to see that any two cyclic-Schottky groups of the same type are quasiconformally conjugated. In particular, the quasiconformal deformation space of a cyclic-Schottky groups of a fixed type (which is connected from the measurable Riemann mapping's theorem) contains all cyclic-Schottky groups of such a type.

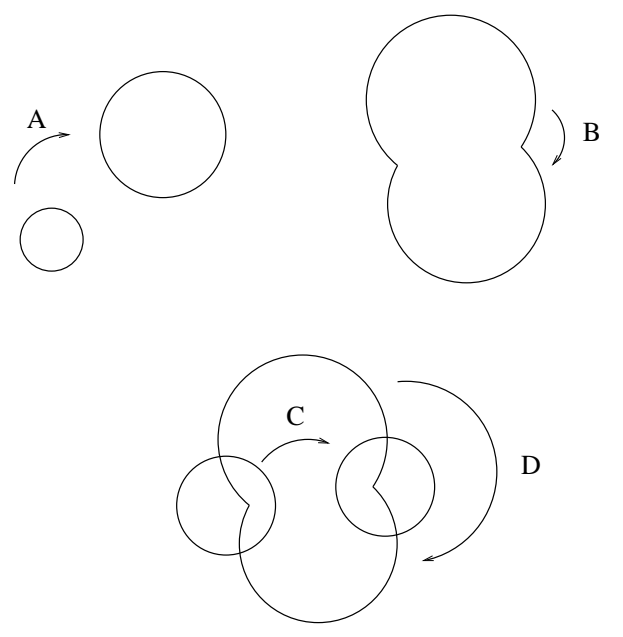

Figure 1. $t=1, r=1, s=1$. 
Let $F(g, p ; t, r, s)$ be the subset of $\mathcal{B}_{g}$ consisting of those $[\Gamma] \in \mathcal{S}_{g}$ for which there exists some $\Gamma_{0} \in[\Gamma]$ and a cyclic-Schottky group $K$, of type $(g, p ; t, r, s)$, containing $\Gamma_{0}$ as an index $p$ normal subgroup.

Let $K$ be a cyclic-Schottky group of type $(g, p ; t, r, s)$ and let $\Gamma$ be a Schottky group of rank $g$ which is an index $p$ normal subgroup of $K$. This provides an embedding of the quasiconformal deformation space $\mathcal{Q}(K)$ into the marked Schottky space $\mathcal{M} S_{g}$, which is projected under $\pi$ to a connected subset of $\mathcal{B}_{g}$ contained inside $F(g, p ; t, r, s)$.

As the cyclic-Schottky group $K$ may contain different Schottky groups of rank $g$ (as index $p$ normal subgroups), it may be that $F(g, p ; t, r, s)$ is disconnected.

In [10] we proved that if $p=2$, then any two Schottky subgroups of index two inside $K$ are quasiconformally conjugated by a quasiconformal homeomorphism that self-conjugates $K$. It follows that $F(g, 2 ; t, r, s)$ is always connected. This is a key observation for our arguments.

\section{Connectivity of $\mathcal{B}_{2}$}

We prove Theorem 2 for $g=2$, that is, that $\mathcal{B}_{2}$ consists of two connected components. These two components will be $F(2,2 ; 1,1,0)$ and $F(2,2 ; 0,1,1)$, both of complex dimension 1.

5.1. Hyperelliptic involution. If $(\Gamma, A, B)$ is a marked Schottky group of rank two, then $E=A B-B A$ is a Möbius transformation of order two so that $E A E=A^{-1}$ and $E B E=B^{-1}$. It follows that $(\Gamma, A, B)$ and $\left(\Gamma, A^{-1}, B^{-1}\right)$ are equivalent marked Schottky groups. The Möbius transformation $E$ induces an automorphism of order two on $M_{\Gamma}$ so that its action on the conformal boundary is the hyperelliptic involution. We call such an involution the hyperelliptic involution on $M_{\Gamma}$; it has exactly three pairwise disjoint simple arcs of fixed points. Due to this, the branch locus $\mathcal{B}_{2}$ corresponds to those conjugacy classes of Schottky groups $\Gamma$ of rank two for which there is a Kleinian group $W$ containing it strictly as a finite index normal subgroup so that $W / \Gamma$ contains elements different from the identity and the hyperelliptic involution.

5.2. Maximal components. Let $W$ be a Kleinian group containing a Schottky group $\Gamma$ (of rank 2) as a finite index normal subgroup so that $W / \Gamma$ contains elements different from the identity and the hyperelliptic involution. Let us consider the natural surjective homomorphism $\theta: W \rightarrow W / \Gamma$. If we consider any element $\rho \in W / \Gamma$ of prime order $p$, different from the hyperelliptic involution, then $K=\theta^{-1}(\langle\rho\rangle)$ is a Kleinian group containing $\Gamma$ as a normal subgroup of index $p$ so that $K / \Gamma$ is a cyclic group of order $p$ which does not contain the hyperelliptic involution. In this way, $\mathcal{B}_{2}$ can be seen as the union of the conjugacy classes of Schottky groups of rank 2 which are normal subgroups of prime order of some Kleinian group and whose quotient cyclic group does not contain the hyperelliptic involution.

Let us write $K / \Gamma=\langle\phi\rangle \cong \mathbf{Z}_{p}$. As described in Section $4.2, K$ is a cyclic-Schottky group of type $(2, p ; t, r, s)$, that is, the free product of $t$ cyclic groups generated by loxodromic transformations, $r$ cyclic groups generated by elliptic transformations of order $p$ and $s$ Abelian groups, each one generated by a loxodromic transformation and an elliptic transformation of order $p$ both of them commuting, so that $1=$ $p(t+r+s-1)-r$. Then the connected components of the set of fixed points (if non-empty) of the automorphism $\phi$ consists of $r$ simple arcs and $s$ simple loops. 
If $\Omega$ is the region of discontinuity of $K$, then $\Omega / K$ is an orbifold of genus $t+s$ containing exactly $2 r$ cone points of order $p$. By the Riemann-Hurwitz formula we must have that $t+s \in\{0,1\}$. In this way,

$$
(p, r) \in\{(2,1),(2,3),(3,2)\} .
$$

Observe that the case $(p, r)=(2,1)$ provides two different cases; they are $(t, s)=$ $(0,1)$ and $(t, s)=(1,0)$.

(1) If $(p, r)=(2,3)$, then $\phi$ is the hyperelliptic involution and we obtain a contradiction.

(2) If $(p, r)=(2,1)$ and $(t, s)=(1,0)$, then $\phi$ has exactly one arc of fixed points and the quotient $M_{\Gamma} /\langle\phi\rangle$ is a handlebody of genus one.

(3) If $(p, r)=(2,1)$ and $(t, s)=(0,1)$, then $\phi$ has exactly two components of fixed points, one is a simple loop and the other a simple arc, and the quotient $M_{\Gamma} /\langle\phi\rangle$ is a handlebody of genus one.

(4) If $(p, r)=(3,2)$, the $\phi$ has exactly two arcs of fixed points and the quotient $M_{\Gamma} /\langle\phi\rangle$ is the closed 3-ball.

As a consequence of all the above, we may see that $\mathcal{B}_{2}$ is the union of three connected subsets. One of them, $F(2,2 ; 1,1,0)$, consists of classes of Schottky groups of rank two which are contained as index two subgroups of cyclic-Schottky groups of type $(2,2 ; 1,1,0)$, the other, $F(2,2 ; 0,1,1)$, consists of classes of Schottky groups of rank two which are contained as index two subgroups of cyclic-Schottky groups of type $(2,2 ; 0,1,1)$, and the third one, $F(2,3 ; 0,2,0)$, consists of classes of Schottky groups of rank two which are contained as index two subgroups of cyclic-Schottky groups of type $(2,3 ; 0,2,0)$. This ensures that $\mathcal{B}_{2}$ can at most have three connected components.

5.3. The component $F(2,2 ; 0,1,1)$ is disjoint from $F(2,2 ; 1,1,0)$. Let us assume there is a Schottky group $\Gamma$ of rank two and cyclic-Schottky groups $K_{1}$ of type $(2,2 ; 0,1,1)$ and $K_{2}$ of type $(2,2 ; 1,1,0)$ both containing $\Gamma$ as an index two subgroup. Set $M=M_{\Gamma}$ and $S=S_{\Gamma}$. Let $\phi_{j}$ be the order two automorphism of $M$ induced by $K_{j}$. The involution $\phi_{1}$ has two components of fixed points, one is an arc and the other is a loop. The involution $\phi_{2}$ has only one component of fixed points, that being an arc. On the genus two closed Riemann surface $S$ we have that the induced conformal involutions, which we still calling as $\phi_{1}$ and $\phi_{2}$, each one acts with exactly two fixed points. It can be checked that $\phi_{1} \circ \phi_{2}$ is the hyperelliptic involution. In particular, $\phi_{1}$ and $\phi_{2}$ commute.

Now, we look at the quotient orbifold $\mathcal{O}_{j}=M /\left\langle\phi_{j}\right\rangle$, for $j=1,2$.

The automorphism $\phi_{2}$ induces an automorphism of order two, say $\psi_{2}$, on $\mathcal{O}_{1}$. It can be seen that $\psi_{2}$ has exactly two arcs of fixed points which intersect both components of the branch locus in $\mathcal{O}_{1}$. The quotient $M /\left\langle\phi_{1}, \phi_{2}\right\rangle=\mathcal{O}_{1} /\left\langle\psi_{2}\right\rangle$ is a closed ball with connected branch locus. On the other hand, the automorphism $\phi_{1}$ induces an automorphism of order two, say $\psi_{1}$, on $\mathcal{O}_{2}$. It can be seen that $\psi_{1}$ has exactly two arcs of fixed points and only one of these arcs intersects the branch locus of $\mathcal{O}_{2}$. The quotient $M /\left\langle\phi_{1}, \phi_{2}\right\rangle=\mathcal{O}_{2} /\left\langle\psi_{1}\right\rangle$ is a closed ball with disconnected branch locus. We have obtained a contradiction.

5.4. The set $\boldsymbol{F}(2,3 ; 0,2,0)$ is connected. A cyclic-Schottky group $K$ of type $(2,3 ; 0,2,0)$ contains exactly two normal Schottky groups of index three, say $\Gamma_{1}$ and $\Gamma_{2}$. In order to see this, we consider $K=\left\langle E_{1}, E_{2}\right\rangle$, where $E_{1}$ and $E_{2}$ are 
of order three ( $K$ is the free product of $\left\langle E_{1}\right\rangle$ and $\left\langle E_{2}\right\rangle$ ), and the possible surjective homomorphisms $\theta: K \rightarrow \mathbf{Z}_{3}$. If we set $u=\theta\left(F_{1}\right)$, then we have only two possibilities for $\theta\left(E_{2}\right) \in\left\{u, u^{-1}\right\}$. If $\theta\left(E_{2}\right)=u$, then $\gamma_{1}=\operatorname{ker}(\theta)=\left\langle E_{1} E_{2}^{-1}, E_{1}^{1} E_{2}\right\rangle$, and if $\theta\left(E_{2}\right)=u^{-1}$, then $\Gamma_{2}=\operatorname{ker}(\theta)=\left\langle E_{1} E_{2}, E_{1}^{-1} E_{2}^{-1}\right\rangle$. It is possible to construct a quasiconformal homeomorphism $W$ so that $W E_{1} W^{-1}=E_{1}$ and $W E_{2} W^{-1}=E_{2}^{-1}$ (this can be seen as a rotation in $180^{\circ}$ of a disc containing the two fixed points of $\left.E_{2}\right)$. Then such a $W$ will conjugate $\Gamma_{1}$ to $\Gamma_{2}$. This ensures that $F(2,3 ; 0,2,0)$ is connected.

5.5. The component $F(2,3 ; 0,2,0)$ is contained inside $F(2,2 ; 1,1,0)$. Let us consider a cyclic-Schottky group $K_{1}$ of type $(2,3 ; 0,2,0)$ generated by the elliptic transformations $E_{1}$ and $E_{2}$, both of order three (see Figure 2 where $A=E_{1}$ and $C=E_{2}^{-1}$ ). Let us denote by $a_{j}$ and $b_{j}$ both fixed points of $E_{j}$, for $j=1,2$. There is a unique elliptic transformation of order two, say $B$, so that $B\left(a_{j}\right)=b_{j}$, for $j=1,2$ (see Figure 2). In fact

$$
B(z)=\frac{\left(a_{1} b_{1}-a_{2} b_{2}\right) z+a_{1} a_{2}\left(b_{1}+b_{2}\right)+b_{1} b_{2}\left(a_{1}+a_{2}\right)}{\left(a_{1}+a_{2}+b_{1}+b_{2}\right) z-a_{1} b_{1}+a_{2} b_{2}} .
$$

It follows that $B E_{j} B^{-1}=E_{j}^{-1}$, for $j=1,2$. The group $K=\left\langle E_{1}, E_{2}, B\right\rangle$ is a Kleinian group. Let us consider the surjective homomorphism $\theta: K \rightarrow\left\langle u, v: u^{3}=\right.$ $\left.v^{2}=(u v)^{2}=1\right\rangle$, defined by $\theta\left(E_{1}\right)=u, \theta\left(E_{2}\right)=u^{-1}$ and $\theta(B)=v$. The kernel of $\theta$ is the Schottky group $\Gamma=\left\langle E_{1} E_{1}, E_{2}^{-1} E_{1}^{-1}\right\rangle$.

The group $\theta^{-1}(\langle u\rangle)$ is $K_{1}$ and the group $\theta^{-1}(\langle v\rangle)$ is generated by $E_{1} E_{2}$ and $B$, which is a cyclic-Schottky group of type $(2,2 ; 1,1,0)$.

All the above ensures that $\mathcal{B}_{2}$ consists of exactly two connected components, one of them is $F(2,2 ; 1,1,0)$ and the other is $F(2,2 ; 0,1,1)$.

Remark 7. The following example clarifies the above construction (up to a quasiconformal conjugation). Let us consider the Möbius transformations $A(z)=$ $E_{1}(z)=e^{2 \pi i / 3} z, B(z)=1 / z$. Then $\langle A, B\rangle$ is a dihedral group of order six. Set $C=B A B=E_{2}^{-1}$, a Möbius transformation of order three so that $\langle B, C\rangle$ is a dihedral group of order six and so that $\langle A, C\rangle$ is a free product (see Figure 2). We may apply Maskit-Klein's combination theorem $[22,24]$ to see that $K=\langle A, B, C\rangle$ is a Kleinian group, with connected region of discontinuity, which is the amalgamated free product, over the cyclic group $\langle B\rangle$, of the groups $\langle A, B\rangle$ and $\langle B, C\rangle$.

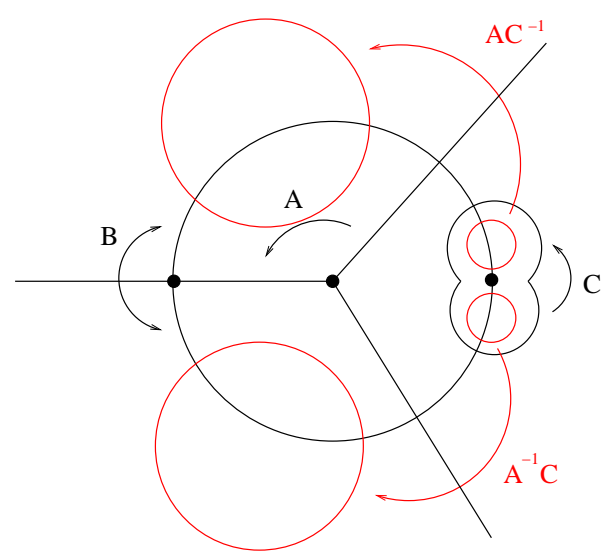

Figure 2. The Kleinian group $K=\langle A, B, C\rangle \cong\langle A, B\rangle *\langle B\rangle\langle B, C\rangle \cong D_{3} * \mathbf{z}_{2} D_{3}$ and the Schottky group $\Gamma=\left\langle A C^{-1}, A^{-1} C\right\rangle$. 
Remark 8. (1) The above asserts that if $M$ is a handlebody, with Kleinian structure provided by a Schottky group $\Gamma_{0}$ of rank two, and $\eta$ is an automorphism of order three (whose fixed point set consists of two simple arcs), then $M$ also admits an automorphism $\rho$ of order two with fixed locus a simple arc. In fact, this is consequence of the fact that given any two Möbius transformations of order three, say $E$ and $F$, then there is a Möbius transformation of order two conjugating $E$ into $F$, and the decomposition structure provided in [13].

(2) Let $K$ be constructed as in Section 5.5. It is not difficult to see that there is a Möbius transformation $D$ of order two commuting with $B$ so that $D A D^{-1}=$ $C$. Again, by Maskit-Klein combination theorem $[22,24]$ the new group $\widehat{K}=$ $\langle A, B, D\rangle \cong D_{2} *_{\mathbf{z}_{2}} D_{3}$ (where $D_{3}=\langle A, B\rangle, D_{2}=\langle B, D\rangle$ and $\mathbf{Z}_{2}=\langle B\rangle$ ) is a Kleinian group for which $\Gamma$ is also a normal subgroup (of index 12).

\section{Connectivity of $\mathcal{B}_{g}$, for $g \geq 3$}

If $g \geq 3$, then generically a handlebody with a Kleinian structure has no hyperelliptic involution. In this situation, $\mathcal{B}_{g}$ consists of those conjugacy classes of Schottky groups of rank $g$ which are strictly contained in a Kleinian group as a finite index normal subgroup (equivalently, the handlebody uniformized by the Schottky group has a non-trivial automorphism).

Lemma 9. The branch locus $\mathcal{B}_{g}$ is the union of the subsets $F(g, p ; t, r, s)$, where $p$ is prime, $t, r, s$ are non-negative integers so that $g-1=p(t+r+s-1)-r$.

Proof. Let $W$ be a Kleinian group containing a Schottky group $\Gamma$ as a non-trivial finite index normal subgroup and let us consider the natural surjective homomorphism $\theta: W \rightarrow W / \Gamma$. Let $\phi \in W / \Gamma$ an element of prime order $p$. The group $K=\theta^{-1}(\langle\phi\rangle)$ is a Kleinian group containing $\Gamma$ as a normal subgroup of index $p$.

Lemma 10. Each $F(g, p ; t, r, s)$, where $p \geq 3$ is prime, intersects some $F(g, 2$; $\left.t^{\prime}, r^{\prime}, s^{\prime}\right)$.

Proof. Let $K$ be a cyclic-Schottky group of type $(g, p ; t, r, s)$, where $p \geq 3$ is a prime.

We consider a surjective homomorphism $\theta: K \rightarrow \mathbf{Z}_{p}=\langle\phi\rangle$ for which $\Gamma=\operatorname{ker}(\theta)$.
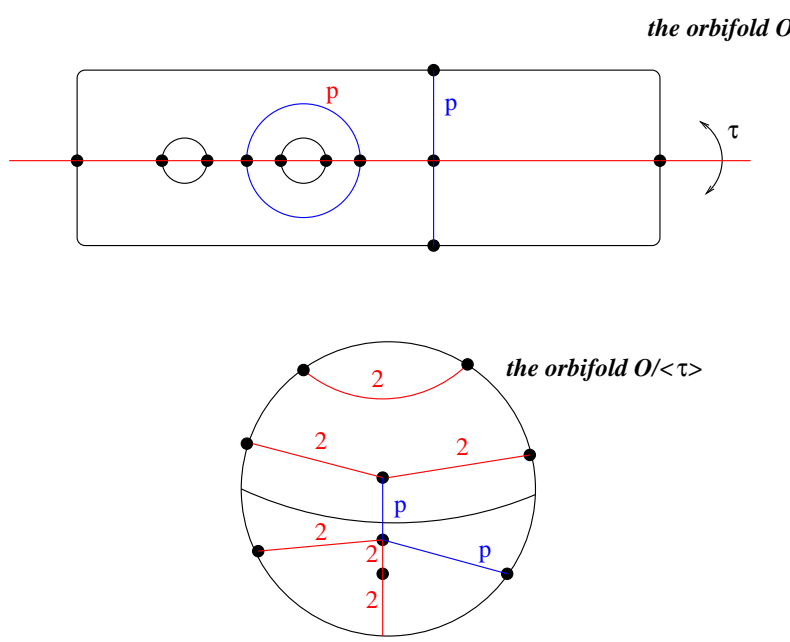

Figure 3. $t=1, r=1, s=1$. 
As described in Section 4.2, the group $K$ is a free product, in the sense of the Klein-Maskit combination theorems, of $t$ cyclic groups generated by loxodromic transformations, $r$ cyclic groups generated by elliptic transformations of order $p$ and $s$ Abelian groups, each one generated by a loxodromic transformation and an elliptic transformation of order $p$ both of them commuting, so that $g=1+p(t+r+s-1)-r$.

The orbifold $\mathcal{O}=M /\langle\phi\rangle$ admits an orientation-preserving self-homeomorphism $\tau$ of order two with the following properties (see Figure 3):

(1) each arc in the branch locus is kept invariant under $\tau$, but $\tau$ permutes the end points of the arc;

(2) each loop in the branch locus is kept invariant under $\tau$ and $\tau$ has exactly two fixed points on the loop.

The involution $\tau$ lifts to a self-homeomorphism $\widehat{\tau}$ (again of order two) of $M$ so that $H=\langle\phi, \widehat{\tau}\rangle \cong D_{p}$ (the dihedral group of order $2 p$ ). In fact, let us identify the orbifold fundamental group of the orbifold $\mathcal{O}$ with $K$. Next, we look at the homotopical action of $\tau$ over $K$. If $K^{\prime}$ denotes the derived subgroup of $K$, then $\tau$ induces the isomomorphism

$$
K / K^{\prime} \rightarrow K / K^{\prime}: k K^{\prime} \mapsto k^{-1} K^{\prime} .
$$

As the branched cover $M \rightarrow \mathcal{O}$ is Abelian (in fact cyclic), we have that $K^{\prime} \triangleleft \Gamma$. It follows that the homotopical action of $\tau$ keeps $\Gamma$ invariant, providing the lifting property. As $\tau$ has order two and it has fixed points, we can find a lifting $\widehat{\tau}$ of order two. The fact that $\widehat{\tau} \circ \phi \circ \widehat{\tau}=\phi^{-1}$ is clear.

In this way, we have that the handlebody $M$ admits the group $H=\langle\phi, \widehat{\tau}\rangle$ as a finite group of orientation-preserving homeomorphisms. It is known that there is a Kleinian structure on $M$ for which $H$ acts as a group of automorphisms [27]. This can also be easily seen as follows: Look at the boundary $S$ of $M$ and the action of $H$ on $S$ as a finite group. By classical results on Riemann surfaces due to Nielsen (see [18]), there is a Riemann surface structure on $S$ so that $H$ is a group of conformal automorphisms. The Riemann surface structure on $S$ induces a Kleinian structure on $M$ for which $H$ acts as a group of automorphisms (see [20, 27]).

It follows that there is a quasiconformal deformation of the group $K$, say $\chi_{\mu}: K \rightarrow$ $K_{\mu}$, so that the group $H$ acts a group of automorphisms of the handlebody with Kleinian structure provided by the corresponding quasiconformal deformation $\chi_{\mu}(\Gamma)=$ $\Gamma_{\mu}$.

We have proved that every $\pi$-image of an embedding of a quasiconformal deformation space of a Kleinian group $K$ containing a Schottky group $\Gamma$ of index a prime $p$ must intersect the $\pi$-image of an embedding of a quasiconformal deformation space of a Kleinian group containing a Schottky group of index two.

As a direct consequence of the both two lemmas is the following fact.

Proposition 11. The number of connected components of the union of all the connected subsets $F(g, 2 ; t, r, s)$ is an upper bound on the number of connected components of $\mathcal{B}_{g}$.

Remark 12. The complex dimension of the subsets $F(g, 2 ; t, r, s)$ is $(3 g-3+r) / 2$. A cyclic-Schottky group of type $(g, 2 ; t, r, s)$ is called a Whittaker group of rank $g$ in [10]. A cyclic-Schottky group of type $(g, 2 ; 0, g+1,0)$ is also called a hyperelliptic Whittaker group of rank $g$. In this last case, the unique index two subgroup which 
does not contain elliptic elements is a Schottky group of rank $g$, called a hyperelliptic Schottky group [17]. As a consequence of the above proposition, the number of connected components of $\mathcal{B}_{g}$ is bounded above by the number of different topological types of Whittaker groups, that is, bounded above by (see [10])

$$
\frac{1}{2}\left(\left[\frac{g+1}{2}\right]+1\right)\left(\left[\frac{g+1}{2}\right]+2\right) \text {. }
$$

The above is a rude upper bound as we will see.

The following two results (whose proofs are provided in the following two sections) imply that $\mathcal{B}_{g}$ consists at most of two connected components $g \geq 3$ and, that $\mathcal{B}_{g}$ is connected for odd $g \geq 3$.

Theorem 13. If $g \geq 4$ is even, then the following hold.

(1) $F(g, 2 ; t, r, s) \cap F(g, 2 ; g / 2,1,0) \neq \emptyset$, if $s$ and $t$ are even.

(2) $F(g, 2 ; t, r, s) \cap F(g, 2 ;(g-2) / 2,3,0) \neq \emptyset$, if $s$ is even and $t$ is odd.

(3) $F(g, 2 ; t, r, s) \cap F(g, 2 ;(g-2) / 2,1,1) \neq \emptyset$, if $s$ is odd and $t$ is even.

(4) $F(g, 2 ; t, r, s) \cap F(g, 2 ;(g-4) / 2,3,1) \neq \emptyset$, if $s$ and $t$ are odd.

(5) $F(g, 2 ; g / 2,1,0) \cap F(g, 2 ;(g-2) / 2,3,0) \neq \emptyset$.

(6) $F(g, 2 ;(g-2) / 2,1,1) \cap F(g, 2 ;(g-4) / 2,3,1) \neq \emptyset$.

Theorem 14. If $g \geq 3$ is odd, then the following hold.

(1) $F(g, 2 ; t, r, s) \cap F(g, 2 ;(g-1) / 2,2,0) \neq \emptyset$, if $t$ is even.

(2) $F(g, 2 ; t, r, s) \cap F(g, 2 ;(g-3) / 2,4,0) \neq \emptyset$, if $t$ is odd.

(3) $F(g, 2 ;(g-1) / 2,2,0) \cap F(g, 2 ;(g-3) / 2,4,0) \neq \emptyset$.

We will work out in details the case $g$ even; as for the case $g$ odd, the constructions are similar; we only indicates the suitable modifications.

Remark 15. Observe that the cyclic-Schottky groups of type $(g, 2 ; g / 2,1,0)$ and $(g, 2 ;(g-1) / 2,2,0)$, induce actions on the Riemann surface $S_{\Gamma}=\Omega / \Gamma$. These induced actions are the same that the ones found in [5] to show that all the Riemann surfaces admitting an action of order 2 or 3 belong to the same connected component of the branch locus of moduli space of Riemann surfaces of genus $g \geq 3$.

\section{Proof of Theorem 13}

If $g$ is even and $K$ is a cyclic-Schottky group of type $(g, 2 ; t, r, s)$, then $r$ is necessarily odd as $g=1+2(t+r+s-1)-r$. So we may write $r=2 b-1$.

In order to prove our theorem, in each case we need to construct a Kleinian group $\widehat{K}$ containing two cyclic-Schottky groups, one of type $(g, 2 ; t, r, s)$ and other of the required type, both of them containing the same Schottky group $\Gamma$ of rank $g$ as index two subgroup.

7.1. Case $t$ and $s$ are even. Let us consider non-negative integers $a, b, c$, so that $t=2 a, r=2 b-1$ and $s=2 c$, and consider a collection of circles as shown in Figure 4; finally, let $\mathcal{D}$ be the common domain bounded by them. Let $F_{1}$ elliptic of order two with both fixed points on $\Sigma_{0}, E_{j}$ elliptic of order two with both fixed points on $\Sigma_{j}$ (for $\left.j=1, \ldots, b\right), A_{j}$ loxodromic transformation so that $A_{j}\left(\Sigma_{1, j, 1}\right)=\Sigma_{1, j, 2}$ and $A_{j}(\mathcal{D}) \cap \mathcal{D}=\emptyset($ for $j=1, \ldots, a), B_{j}$ loxodromic transformation so that $B_{j}\left(\Sigma_{2, j, 1}\right)=\Sigma_{2, j, 2}$ and $B_{j}(\mathcal{D}) \cap \mathcal{D}=\emptyset$ (for $\left.j=1, \ldots, c\right), C_{j}$ elliptic of order two with both fixed points on $\Sigma_{2, j, 3}$ and commuting with $B_{j}$ (for $j=1, \ldots, c$ ). 
Let $\widehat{K}$ be generated by all the above Möbius transformations. Then $\widehat{K}$ turns out to be a Kleinian group (by the Klein-Maskit combination theorems) with $\mathcal{D}$ as a fundamental domain, isomorphic to the free product of a copy of $\mathbf{Z}_{2}^{2}$ (the group generated by $F_{1}$ and $E_{1}$ ), $b-1$ copies of $\mathbf{Z}_{2}$ (each one generated by $E_{2}, \ldots, E_{b}$, respectively), $a$ infinite cyclic groups (generated by $A_{1}, \ldots, A_{a}$, respectively), and $c$ copies of $\mathbf{Z} \times \mathbf{Z}_{2}$ (generated by $B_{j}$ and $C_{j}$, for $j=1, \ldots, c$ ).

Moreover, by the same combination theorems, if $\Omega$ is the region of discontinuity of $\widehat{K}$, then $\Omega$ is connected (the complement of a Cantor set) and $\Omega / \widehat{K}$ is of signature $(a+c ; 2,2 b-1,2)$.

Let us consider the surjective homomorphism

$$
\theta: \widehat{K} \rightarrow\left\langle u, v: u^{2}=v^{2}=(u v)^{2}=1\right\rangle \cong \mathbf{Z}_{2}^{2}
$$

defined by

$$
\begin{aligned}
& \theta\left(E_{1}\right)=\cdots=\theta\left(E_{b}\right)=\theta\left(C_{1}\right)=\cdots=\theta\left(C_{c}\right)=u, \quad \theta\left(F_{1}\right)=v \\
& \theta\left(A_{1}\right)=\cdots=\theta\left(A_{a}\right)=\theta\left(B_{1}\right)=\cdots=\theta\left(B_{c}\right)=1 .
\end{aligned}
$$

The kernel of $\theta$ is a torsion free Kleinian group $\Gamma$; which is a normal subgroup of index 4 in $\widehat{K}$. As $\Gamma$ has also $\Omega$ as its region of discontinuity, it follows from the classification of function groups that $\Gamma$ is necessarily a Schottky group. Its rank $g$ can be computed by the Riemann-Hurwitz formula, i.e., $g=4 a+2 b+4 c-2$.

The group $K_{1}=\theta^{-1}(\langle u\rangle)$ is generated by the transformations

$$
\begin{aligned}
& E_{1}, \ldots, E_{b}, C_{1}, \ldots, C_{s}, F_{1} E_{2} F_{1}, \ldots, F_{1} E_{b} F_{1}, F_{1} C_{1} F_{1}, \ldots, F_{1} C_{c} F_{1}, \\
& A_{1}, \ldots, A_{a}, B_{1}, \ldots, B_{c}, F_{1} A_{1} F_{1}, \ldots, F_{1} A_{a} F_{1}, F_{1} B_{1} F_{1}, \ldots, F_{1} B_{c} F_{1}
\end{aligned}
$$

and it is cyclic-Schottky group of type $(g, 2 ; 2 a, 2 b-1,2 c)$. The group $K_{2}=\theta^{-1}(\langle v\rangle)$ is generated by the transformations

$$
F_{1}, E_{1} E_{2}, \ldots, E_{1} E_{b}, E_{1} C_{1}, \ldots, E_{1} C_{c}, A_{1}, \ldots, A_{a}, B_{1}, \ldots, B_{c}, E_{1} A_{1} E_{1}, \ldots, E_{1} A_{a} E_{1}
$$

and it is cyclic-Schottky group of type $(g, 2 ; 2 a+b+2 c-1,1,0)$.

The group $K_{1}$ induces an involution on $M_{\Gamma}$ whose branch locus consists of exactly $2 c$ loops and $2 b-1$ arcs and the group $K_{2}$ induces an involution on $M_{\Gamma}$ whose branch locus consists of exactly 1 arc of fixed points.
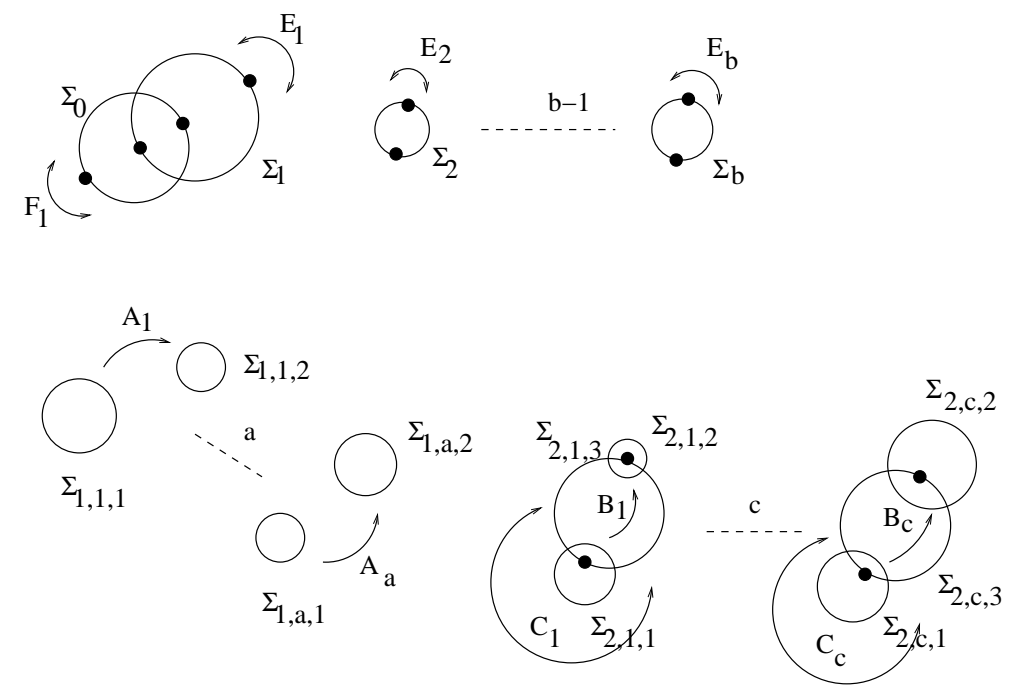

Figure 4. The Kleinian group $\widehat{K}$ for $g$ even and $t=2 a, r=2 b-1$ and $s=2 c$. 

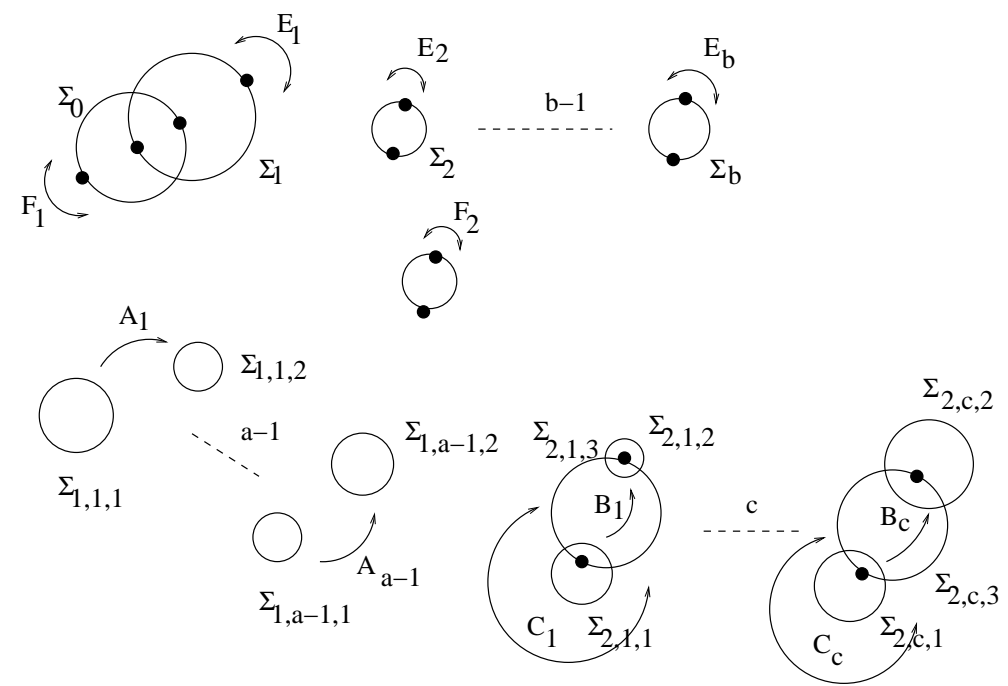

Figure 5. The Kleinian group $\widehat{K}$ for $g$ even and $t=2 a-1, r=2 b-1$ and $s=2 c$.

7.2. Case $t$ odd and $s$ even. If $t=2 a-1$, then we consider a Kleinian group $\widehat{K}$ as in the previous section, but we delete the transformation $A_{a}$ and add an elliptic element of order two, say $F_{2}$. We use the same homomorphism $\theta$ as before with $\theta\left(F_{2}\right)=v$. Then $K_{1}=\theta^{-1}(\langle u\rangle)$ will be a cyclic-Schottky group of type $(g, 2 ; t, r, s)$ and $K_{2}=\theta^{-1}(\langle v\rangle)$ will be a cyclic-Schottky group of type $(g, 2 ;(g-2) / 2,3,0)$.
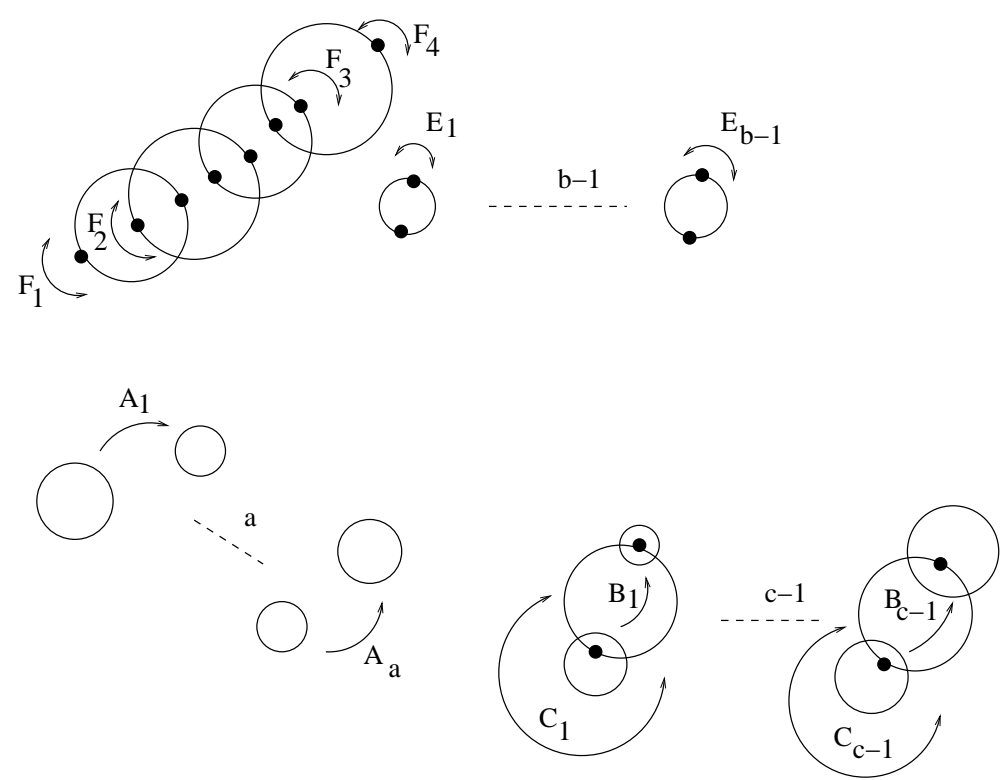

Figure 6. The group $\widehat{K}$ for $g$ even and $t$ odd.

7.3. Case $\boldsymbol{s}$ odd and $\boldsymbol{t}$ even. Let us write $s=2 c-1$ and $t=2 a$. Then $\widehat{K}$ is the Kleinian group constructed a the free product of $(b-1)$ cyclic groups generated by elliptic elements $E_{1}, \ldots, E_{b-1}$ of order $2, a$ cyclic groups generated by loxodromic transformations $A_{1}, \ldots, A_{a},(c-1)$ groups isomorphic to $\mathbf{Z} \times \mathbf{Z}_{2}$ generated by the loxodromic transformations $B_{1}, \ldots, B_{c-1}$ and the elliptic transformations of order two $C_{1}, \ldots, C_{c-1}$, so that $B_{j} C_{j}=C_{j} B_{j}$, and a Kleinian group generated by elliptic transformations of order two $F_{1}, F_{2}, F_{3}$ and $F_{4}$, so that $F_{1} F_{2}=F_{2} F_{1}, F_{2} F_{3}=F_{3} F_{2}$ and $F_{3} F_{4}=F_{4} F_{3}$ (see Figure 6 ). In this case we consider the surjective homomorphism 
$\theta: \widehat{K} \rightarrow\langle u, v\rangle \cong \mathbf{Z}_{2}^{2}$, defined by

$$
\begin{aligned}
& \theta\left(E_{j}\right)=\theta\left(C_{k}\right)=\theta\left(F_{2}\right)=\theta\left(F_{4}\right)=u, \\
& \theta\left(F_{1}\right)=\theta\left(F_{3}\right)=v, \quad \theta\left(A_{l}\right)=\theta\left(B_{k}\right)=1 .
\end{aligned}
$$

Then $K_{1}=\theta^{-1}(\langle u\rangle)$ is a cyclic-Schottky group of type $(g, 2 ; t, r, s)$ and $K_{2}=$ $\theta^{-1}(\langle v\rangle)$ is of type $(g, 2 ;(g-2) / 2,1,1)$.

7.4. Case $s$ and $\boldsymbol{t}$ odd. Let us write $s=2 c-1$ and $t=2 a-1$. Then $\widehat{K}$ is the Kleinian group constructed a the free product of $b$ cyclic groups generated by elliptic elements $E_{1}, \ldots, E_{b-1}, F_{5}$ of order $2,(a-1)$ cyclic groups generated by loxodromic transformations $A_{1}, \ldots, A_{a-1},(c-1)$ groups isomorphic to $\mathbf{Z} \times \mathbf{Z}_{2}$ generated by the loxodromic transformations $B_{1}, \ldots, B_{c-1}$ and the elliptic transformations $C_{1}, \ldots, C_{c-1}$ of order two, so that $B_{j} C_{j}=C_{j} B_{j}$, and a Kleinian group generated by elliptic transformations of order two, $F_{1}, F_{2}, F_{3}$ and $F_{4}$, so that $F_{1} F_{2}=F_{2} F_{1}, F_{2} F_{3}=F_{3} F_{2}$ and $F_{3} F_{4}=F_{4} F_{3}$ (see Figure 7 ). In this case we consider the surjective homomorphism $\theta: \widehat{K} \rightarrow\langle u, v\rangle \cong \mathbf{Z}_{2}^{2}$, defined by

$$
\begin{aligned}
& \theta\left(E_{j}\right)=\theta\left(C_{k}\right)=\theta\left(F_{2}\right)=\theta\left(F_{4}\right)=u, \\
& \theta\left(F_{1}\right)=\theta\left(F_{3}\right)=\theta\left(F_{5}\right)=v, \quad \theta\left(A_{l}\right)=\theta\left(B_{k}\right)=1 .
\end{aligned}
$$

Then $K_{1}=\theta^{-1}(\langle u\rangle)$ is a cyclic-Schottky group of type $(g, 2 ; t, r, s)$ and $K_{2}=$ $\theta^{-1}(\langle v\rangle)$ is of type $(g, 2 ;(g-4) / 2,3,1)$.

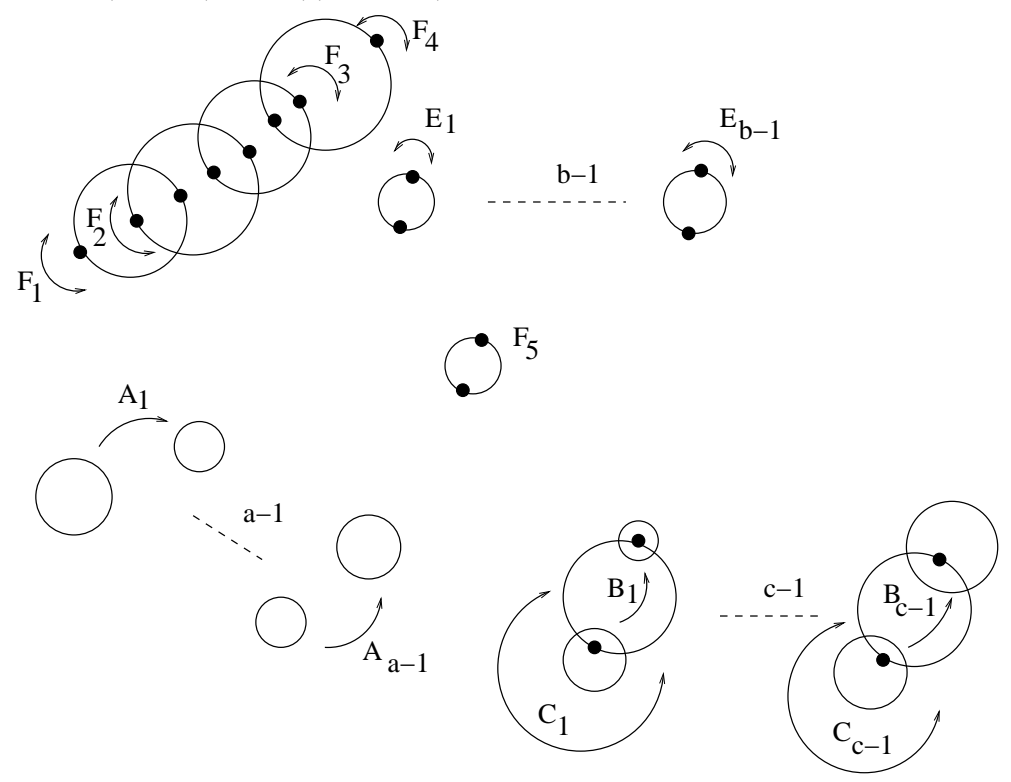

Figure 7. The group $\widehat{K}$ for $g$ even and $s$ and $t$ odd.

7.5. $\boldsymbol{F}(g, 2 ; g / 2,1,0) \cap \boldsymbol{F}(g, 2 ;(g-2) / 2,3,0) \neq \emptyset$. Let us consider a Kleinian group which is the free product of $g / 2$ cyclic groups of order two, say generated by the elliptic elements $F_{1}, \ldots, F_{g / 2}$, and a group isomorphic to $\mathbf{Z}_{2}^{2}$ generated by the elliptic elements $E_{1}$ and $E_{2}$ (see Figure 8 ). Now consider the surjective homomorphism $\theta: \widehat{K} \rightarrow\langle u, v\rangle \cong \mathbf{Z}_{2}^{2}$, defined by

$$
\theta\left(F_{1}\right)=\cdots=\theta\left(F_{(g-2) / 2}\right)=u v, \quad \theta\left(E_{1}\right)=u, \quad \theta\left(E_{2}\right)=\theta\left(F_{g / 2}\right)=v .
$$

Then $K_{1}=\theta^{-1}(\langle u\rangle)$ is a cyclic-Schottky group of type $(g, 2 ; g / 2,1,0)$ and $K_{2}=$ $\theta^{-1}(\langle v\rangle)$ is of type $(g, 2 ;(g-2) / 2,3,0)$. 

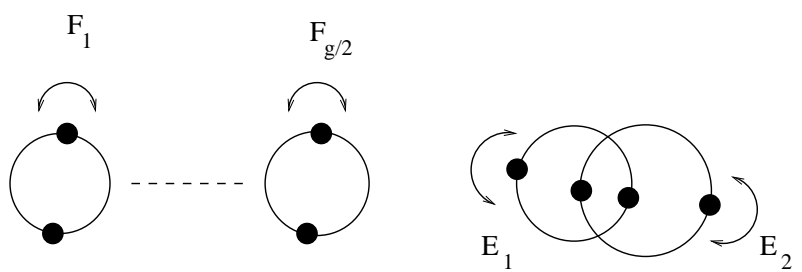

Figure 8. A group $\widehat{K}$.

7.6. $F(g, 2 ;(g-2) / 2,1,1) \cap F(g, 2 ;(g-4) / 2,3,1) \neq \emptyset$. Let us consider a Kleinian group which is the free product of $(g-2) / 2$ cyclic groups of order two generated by the elliptic elements $F_{1}, \ldots, F_{(g-2) / 2}$, and a group generated by the elliptic elements of order two $E_{1}, E_{2}, E_{3}$ and $E_{4}$, so that $E_{1} E_{2}=E_{2} E_{1}, E_{3}=$ $E_{3} E_{2}$ and $E_{3} E_{4}=E_{4} E_{3}$ (see Figure 9 ). Now consider the surjective homomorphism $\theta: \widehat{K} \rightarrow\langle u, v\rangle \cong \mathbf{Z}_{2}^{2}$, defined by

$$
\begin{aligned}
& \theta\left(F_{1}\right)=\cdots=\theta\left(F_{(g-4) / 2}\right)=u v, \\
& \theta\left(E_{1}\right)=\theta\left(E_{3}\right)=\theta\left(F_{(g-2) / 2}\right)=v, \quad \theta\left(E_{2}\right)=\theta\left(E_{3}\right)=u .
\end{aligned}
$$

Then $K_{1}=\theta^{-1}(\langle u\rangle)$ is a cyclic-Schottky group of type $(g, 2 ;(g-2) / 2,1,1)$ and $K_{2}=\theta^{-1}(\langle v\rangle)$ is of type $(g, 2 ;(g-4) / 2,3,1)$.
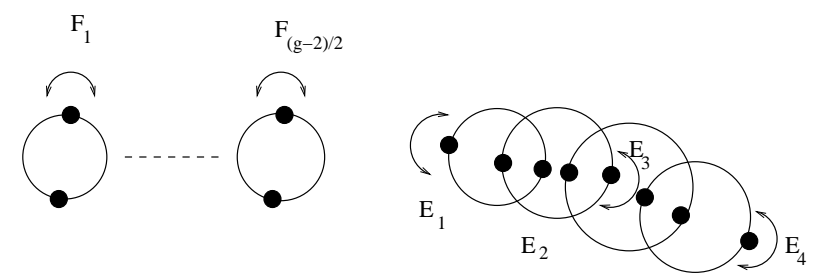

Figure 9. A group $\widehat{K}$.

\section{Proof of Theorem 14}

If $g$ is odd and $K$ is a cyclic-Schottky group of type $(g, 2 ; t, r, s)$, then $r$ is necessarily even as $g=1+2(t+r+s-1)-r$. So we may write $r=2 b$.

8.1. Case $s$ and $\boldsymbol{t}$ even. Let us write $s=2 c$ and $t=2 a$. Then $\widehat{K}$ is the Kleinian group constructed a the free product of $(b+1)$ cyclic groups generated by elliptic elements $E_{1}, \ldots, E_{b}$ and $F_{1}$ of order $2, a$ cyclic groups generated by loxodromic transformations $A_{1}, \ldots, A_{a}$, and $c$ groups isomorphic to $\mathbf{Z} \times \mathbf{Z}_{2}$ generated by the loxodromic transformations $B_{1}, \ldots, B_{c}$ and the elliptic transformations $C_{1}, \ldots, C_{c}$ of order two, so that $B_{j} C_{j}=C_{j} B_{j}$. In this case we consider the surjective homomorphism $\theta: \widehat{K} \rightarrow\langle u, v\rangle \cong \mathbf{Z}_{2}^{2}$, defined by

$$
\theta\left(E_{j}\right)=\theta\left(C_{k}\right)=u, \quad \theta\left(F_{1}\right)=v, \quad \theta\left(A_{l}\right)=\theta\left(B_{k}\right)=1 .
$$

Then $K_{1}=\theta^{-1}(\langle u\rangle)$ is a cyclic-Schottky group of type $(g, 2 ; t, r, s)$ and $K_{2}=$ $\theta^{-1}(\langle v\rangle)$ is of type $(g, 2 ;(g-1) / 2,2,0)$.

8.2. Case $s$ is even and $\boldsymbol{t}$ is odd. Let us write $s=2 c$ and $t=2 a-1$. Then $\widehat{K}$ is the Kleinian group constructed a the free product of $(b+2)$ cyclic groups generated by elliptic elements $E_{1}, \ldots, E_{b}, F_{1}$ and $F_{2}$ of order $2,(a-1)$ cyclic groups generated by loxodromic transformations $A_{1}, \ldots, A_{a-1}$, and $c$ groups isomorphic to $\mathbf{Z} \times \mathbf{Z}_{2}$ generated by the loxodromic transformations $B_{1}, \ldots, B_{c}$ and the elliptic transformations 
$C_{1}, \ldots, C_{c}$ of order two so that $B_{j} C_{j}=C_{j} B_{j}$. In this case we consider the surjective homomorphism $\theta: \widehat{K} \rightarrow\langle u, v\rangle \cong \mathbf{Z}_{2}^{2}$, defined by

$$
\theta\left(E_{j}\right)=\theta\left(C_{k}\right)=u, \quad \theta\left(F_{1}\right)=\theta\left(F_{2}\right)=v, \quad \theta\left(A_{l}\right)=\theta\left(B_{k}\right)=1 .
$$

Then $K_{1}=\theta^{-1}(\langle u\rangle)$ is a cyclic-Schottky group of type $(g, 2 ; t, r, s)$ and $K_{2}=$ $\theta^{-1}(\langle v\rangle)$ is of type $(g, 2 ;(g-3) / 2,4,0)$.

8.3. Case $\boldsymbol{s}$ is odd and $\boldsymbol{t}$ is even. Let us write $s=2 c-1$ and $t=2 a$. Then $\widehat{K}$ is the Kleinian group constructed a the free product of $b$ cyclic groups generated by elliptic elements $E_{1}, \ldots, E_{b}$ of order $2, a$ cyclic groups generated by loxodromic transformations $A_{1}, \ldots, A_{a},(c-1)$ groups isomorphic to $\mathbf{Z} \times \mathbf{Z}_{2}$ generated by the loxodromic transformations $B_{1}, \ldots, B_{c-1}$ and the elliptic transformations $C_{1}, \ldots, C_{c-1}$ of order two, so that $B_{j} C_{j}=C_{j} B_{j}$, and a group generated by three elliptic elements of order two: $F_{1}, F_{2}$ and $F_{3}$, so that $F_{1} F_{2}=F_{2} F_{1}$ and $F_{2} F_{3}=F_{3} F_{2}$. In this case we consider the surjective homomorphism $\theta: \widehat{K} \rightarrow\langle u, v\rangle \cong \mathbf{Z}_{2}^{2}$, defined by

$$
\theta\left(E_{j}\right)=\theta\left(C_{k}\right)=\theta\left(F_{2}\right)=u, \quad \theta\left(F_{1}\right)=\theta\left(F_{3}\right)=v, \quad \theta\left(A_{l}\right)=\theta\left(B_{k}\right)=1 .
$$

Then $K_{1}=\theta^{-1}(\langle u\rangle)$ is a cyclic-Schottky group of type $(g, 2 ; t, r, s)$ and $K_{2}=$ $\theta^{-1}(\langle v\rangle)$ is of type $(g, 2 ;(g-1) / 2,2,0)$.

8.4. Case $s$ and $t$ odd. Let us write $s=2 c-1$ and $t=2 a-1$. Then $\widehat{K}$ is the Kleinian group constructed a the free product of $(b+1)$ elliptic elements of order 2 , say $E_{1}, \ldots, E_{b}$ and $F_{1},(a-1)$ cyclic groups generated by loxodromic transformations, say $A_{1}, \ldots, A_{a-1},(c-1)$ groups isomorphic to $\mathbf{Z} \times \mathbf{Z}_{2}$, say generated by the loxodromic transformations $B_{1}, \ldots, B_{c-1}$ and the elliptic transformations of order two $C_{1}, \ldots, C_{c-1}$, so that $B_{j} C_{j}=C_{j} B_{j}$, and a group generated by three elliptic elements of order two: $F_{2}, F_{3}$ and $F_{4}$, so that $F_{2} F_{3}=F_{3} F_{2}$ and $F_{3} F_{4}=F_{4} F_{3}$. In this case we consider the surjective homomorphism $\theta: \widehat{K} \rightarrow\langle u, v\rangle \cong \mathbf{Z}_{2}^{2}$, defined by

$$
\theta\left(E_{j}\right)=\theta\left(C_{k}\right)=\theta\left(F_{3}\right)=u, \quad \theta\left(F_{1}\right)=\theta\left(F_{2}\right)=\theta\left(F_{4}\right)=v, \quad \theta\left(A_{l}\right)=\theta\left(B_{k}\right)=1 .
$$

Then $K_{1}=\theta^{-1}(\langle u\rangle)$ is a cyclic-Schottky group of type $(g, 2 ; t, r, s)$ and $K_{2}=$ $\theta^{-1}(\langle v\rangle)$ is of type $(g, 2 ;(g-3) / 2,4,0)$.

8.5. $\boldsymbol{F}(g, 2 ;(g-1) / 2,2,0) \cap \boldsymbol{F}(g, 2 ;(g-3) / 2,4,0) \neq \emptyset$. Let us consider a Kleinian group which is the free product of $(g=3) / 2$ cyclic groups of order two, generated by the elliptic elements $F_{1}, \ldots, F_{(g+1) / 2}$, and $E$. Now consider the surjective homomorphism $\theta: \widehat{K} \rightarrow\langle u, v\rangle \cong \mathbf{Z}_{2}^{2}$, defined by

$$
\theta\left(F_{3}\right)=\cdots=\theta\left(F_{(g+1) / 2}\right)=u v, \quad \theta(E)=u, \quad \theta\left(F_{1}\right)=\theta\left(F_{2}\right)=v .
$$

Then $K_{1}=\theta^{-1}(\langle u\rangle)$ is a cyclic-Schottky group of type $(g, 2 ;(g-1) / 2,2,0)$ and $K_{2}=\theta^{-1}(\langle v\rangle)$ is of type $(g, 2 ;(g-3) / 2,4,0)$.

Acknowledgments. The authors would like to thank the referee for his/her careful reading and the suggested corrections to the first version.

\section{References}

[1] Ahfors, L., and L. Bers: Riemann mapping's theorem for variable metrics. - Ann. of Math. $72,1960,385-404$.

[2] Bartolini, G., A. F. Costa, and M. Izquierdo: On isolated strata of pentagonal Riemann surfaces in the branch locus of moduli spaces. - In: Computational Algebraic and Analytic Geometry, Contemp. Math. 572, 2012, 19-24. 
[3] Bartolini, G., A.F. Costa, and M. Izquierdo: On the connectivity of branch loci of moduli spaces. - Ann. Acad. Sci. Fenn. Math. 38:1, 2013, 245-258.

[4] Bartolini, G., A. F. Costa, M. Izquierdo, and A. M. Porto: On the connectedness of the branch locus of the moduli space of Riemann surfaces. - Rev. R. Acad. Cienc. Exactas Fís. Nat. Ser. A Mat. RACSAM 104:1, 2010, 81-86.

[5] Bartolini, G., and M. Izquierdo: On the connectedness of the branch locus of the moduli space of Riemann surfaces of low genus. - Proc. Amer. Math. Soc. 140:1, 2012, 35-45.

[6] Bers, L.: Spaces of Kleinian groups. - Lecture Notes in Math. 155, Springer, Berlin, 1970, 9-34.

[7] Bers, L.: Automorphic forms for Schottky groups. - Adv. Math. 16, 1975, 332-361.

[8] Bujalance, E., A. F. Costa, and M. Izquierdo: A note on isolated points in the branch locus of the moduli space of compact Riemann surfaces. - Ann. Acad. Sci. Fenn. Math. 23:1, $1998,25-32$.

[9] Chuckrow, V.: On Schottky groups with applications to Kleinian groups. - Ann. of Math. 88, 1968, 47-61.

[10] Díaz, R., I. Garijo, G. Gromadzki, and R. A. Hidalgo: Structure of Whittaker groups and application to conformal involutions on handlebodies. - Topology Appl. 157, 2010, 23472361.

[11] Earle, C. J.: The group of biholomorphic self-mappings of Schottky space. - Ann. Acad. Sci. Fenn. Ser. A I Math. 16:2, 1991, 399-410.

[12] Hidalgo, R. A.: On the 12(g-1) bound. - C. R. Math. Rep. Acad. Sci. Canada 18, 1996, $39-42$.

[13] Hidalgo, R. A.: Cyclic extensions of Schottky uniformizations. - Ann. Acad. Sci. Fenn. Math. 29, 2004, 329-344.

[14] Hidalgo, R. A.: Automorphisms of Schottky type. - Ann. Acad. Sci. Fenn. Math. 30, 2005, 183-204.

[15] Hidalgo, R. A.: Maximal Schottky extension groups. - Geom. Dedicata 146:1, 2010, 141-158.

[16] Hidalgo, R. A.: Maximal virtual Schottky groups: Explicit constructions. - Rev. Colombiana Mat. 44, 2010, 41-57.

[17] Keen, L.: On hyperelliptic Schottky groups. - Ann. Acad. Sci. Fenn. Ser. A I Math. 5, 1980, $165-174$.

[18] Kerckhoff, S.: The Nielsen realization problem. - Ann. of Math. 117, 1983, 235-265.

[19] KULKARNI, R. S.: Isolated points in the branch locus of the moduli space of compact Riemann surfaces. - Ann. Acad. Sci. Fenn. Ser. A I Math. 16, 1991, 71-81.

[20] MeCullough, D., A. Miller, and B. Zimmermann: Group actions on handlebodies. Proc. Lond. Math. Soc. 59, 1989, 373-415.

[21] Maskit, B.: Self-maps of Kleinian groups. - Amer. J. Math. 93, 1971, 840-856.

[22] Maskit, B.: On Klein's combination theorem III. - Ann. of Math. Stud. 66, 1971, 297-316.

[23] Maskit, B.: Kleinian groups. - Grundlehren Math. Wiss. 287, Springer-Verlag, 1988.

[24] Maskit, B.: On Klein's combination theorem. IV. - Trans. Amer. Math. Soc. 336, 1993, 265-294.

[25] Morrey, C. B., JR.: On the solutions of quasi-linear elliptic partial differential equations. Trans. Amer. Math. Soc. 43:1, 1938, 126-166.

[26] NAG, S.: The complex analytic theory of Teichmüller spaces. - A Wiley-Interscience Publication, John Wiley \& Sons, New York, 1988.

[27] Zimmermann, B.: Über Homöomorphismen $n$-dimensionaler Henkelkörper und endliche Erweiterungen von Schottky-Gruppen. - Comment. Math. Helv. 56:3, 1981, 474-486 (in German).

Received 17 July 2013 • Revised received 1 April 2014 • Accepted 4 April 2014 